\title{
Protée
}

\section{Cendres et dessin}

\section{La représentation en ruine chez Derrida}

\section{Joana Masó}

Volume 35, numéro 2, automne 2007

Imaginaire des ruines

URI : https://id.erudit.org/iderudit/017471ar

DOI : https://doi.org/10.7202/017471ar

Aller au sommaire du numéro

\section{Éditeur(s)}

Département des arts et lettres - Université du Québec à Chicoutimi

ISSN

0300-3523 (imprimé)

1708-2307 (numérique)

Découvrir la revue

Citer cet article

Masó, J. (2007). Cendres et dessin : la représentation en ruine chez Derrida. Protée, 35(2), 89-92. https://doi.org/10.7202/017471ar

\section{Résumé de l'article}

Le présent article cherche à exposer la pensée derridienne des ruines comme la condition de possibilité de toute oeuvre, que nous retraçons à partir de deux figures paradigmatiques : la cendre de Feu la cendre et l'autoportrait des dessinateurs dans Mémoires d'aveugle. L'Autoportrait et autres ruines. Nous mettrons en lumière la temporalité impossible de la ruine, son incomplétude originaire ainsi que son invisibilité, qui configurent l'originalité de l'approche derridienne des ruines.
Ce document est protégé par la loi sur le droit d'auteur. L'utilisation des services d'Érudit (y compris la reproduction) est assujettie à sa politique d'utilisation que vous pouvez consulter en ligne.

https://apropos.erudit.org/fr/usagers/politique-dutilisation/ 


\title{
CENDRES ET DESSIN : LA REPRÉSENTATION EN RUINE CHEZ DERRIDA
}

\author{
JOANA MASÓ
}

"Il y a la cendre, mais une cendre n'est pas» (1987: 23) écrit Jacques Derrida dans Feu la cendre, bref texte qui met en scène une pensée des cendres comme les ruines de l'être, ainsi qu'une ruine de l'écriture. Des pages écrites donc qui, à côté des innombrables pages de Feu la cendre qui auraient été brûlées ou incinérées, propagent le leitmotiv de la «restance du reste», autre nom derridien pour la cendre ou la ruine.

Cette ruine qui "n'est pas l'être» (1986: 77), écrit le philosophe, a pour mode de présence le non-être ou $\mathrm{I}^{\prime}$ «imprésence», car loin de s'écrire comme le reste de ce qui fut jadis, la cendre est un reste de ce qui ne fut pas et ne serait donc pas concernée par l'être: «[l]a cendre n'est pas, elle n'est pas ce qui est. Elle reste de ce qui n'est pas, pour ne rappeler au fond friable d'elle que non-être ou imprésence» (1987: 11). "L'être sans présence n'a pas été», continue Derrida dans sa lecture de la cendre; par cette formule, le philosophe rappelle le propre de l'être, à savoir sa présence (ce qui a été, dans le passé, est dans le présent et sera dans l'avenir), en annonçant dans le même temps que tout être qui n'aurait plus affaire à la présence ( «l'être sans présence») n'aurait finalement pas affaire non plus avec l'être (il n'aurait «pas été »). Penser donc l'être de la cendre comme ce qui n'aurait "pas été » briserait ou interromprait l'inaltérabilité du concept métaphysique de présence.

Deux altérations viennent ruiner la présence à soi de l'être dans Feu la cendre: la dissémination et l'impossible appropriation du reste. D'abord, la dissémination derridienne est le mouvement de la « dispersion sans retour [...] qui ne reste pas et ne revient à personne» (ibid.: 23), elle incinère la position (la thesis grecque et la stasis latine), la fixité et donc la coïncidence avec un soi-même par le biais d'incessants et immaîtrisables déplacements. Une dissémination qui, n'étant pas la décision ou le résultat de la maîtrise d'un sujet, se dessine comme une figure de l'ex-appropriation derridienne: nous nous approchons de la ruine de l'être en cherchant à nous en approprier le sens, la signification, la portée et les implications (historiques, philosophiques...), mais tout ce que nous pouvons penser sous le nom de «sens » dépasse inévitablement notre intention d'appropriation, nous reste impropre parce que disséminé et irrécupérable dans sa complétude. La cendre comme ruine de l'être appellerait ainsi, et indissociablement, une volonté d'appropriation et une puissante expropriation qui ne viendrait pas de nous, deux mouvements qui amènent le philosophe à forger le néologisme exappropriation.

Très loin donc de ce qu'Hélène Cixous a appelé des «ruines qui ne sont pas des ruines, mais des hymnes à la mémoire lumineuse» (1986: 54), la cendre qu'«il y a» dans Feu la cendre n'exalte l'être d'aucun passé dont elle rapporterait la vérité, parce qu'elle est un lieu non pas de mémoire mais de perte sans retour; de même, elle porte en soi non pas ce qui a été mais plutôt «la différence entre ce qui reste et ce qui est». C'est ainsi que «la cendre, ce vieux mot gris, ce thème poussiéreux de l'humanité (Derrida, 1987: 53), arrivera à s'écrire comme un autre nom de la trace, la différance ou l'écriture derridiennes: "entre le noir et le blanc, la couleur de l'écriture ressemble à la seule "littéralitée" de la cendre qui tienne encore dans un langage» (ibid.: 33). 
J'ai maintenant l'impression que le meilleur paradigme de la trace n'est pas [...] la piste de chasse, le frayage, le sillon dans le sable, le sillage dans la mer, l'amour du pas pour son empreinte, mais la cendre (ce qui reste sans rester de l'holocauste, du brûle-tout, de l'incendie l'encens). (Ibid. : 27)

Si donc la cendre est à lire comme «le meilleur paradigme de la trace», elle est tout de suite dessinée comme une figure du renvoi sans origine. Certes, comme la trace, la cendre est cendre de, à savoir qu'elle n'est pas énonçable absolument, elle ne peut se couper du renvoi à l'infini, elle renvoie incessamment. Et, tant qu'elle sera empêchée d'interrompre ce renvoi, l'identification avec cela même à quoi elle renvoie s'avérera impossible: «gardant la marque de l'élément passé et se laissant déjà creuser par la marque de son rapport à l'élément futur » (Derrida, 1972: 13), la trace fait différer, elle est l'autre nom du processus derridien du différer comme espacement et intervalle. C'est ainsi que la cendre, n'étant qu'un moment de la différance, «est une cendre encore de la cendre même» (1987: 33).

Comme la différance, la cendre ne serait «ni un nom ni un concept» (1972: 3), car ceux-ci présupposeraient déjà du plein, du fini, du coupé et non pas la chaîne inclôturable de différés. C'est pourquoi nous penserons la «cendre» qui dit ici la ruine sous le mot derridien de faisceau, aconceptuel et anominal, qui exprime l' «intrication», le «tissage», le «croisement» du sens (ibid.: 4). Cette cendre, qui n'est «ni un nom ni un concept» et qui est donc d'une certaine manière silencieuse - elle ne fait pas de la mémoire un drapeau -, est porteuse de témoignage, comme dans le poème de Paul Celan, Aschenglorie (Cendres - La gloire), commenté par Derrida dans Schibboleth. La cendre s'écrirait ici comme un lieu de témoignage sans vérité à vérifier: une cendre « irréductible au concept, au savoir et même à l'histoire, à la tradition » (1986: 77), mais renvoyant à ceux-ci et les renvoyant de manière à faire arriver ce mode de présence qui est celui de la spectralité.

Le long travail derridien sur la spectralité, qui s'inscrit dans le projet de déconstruction de la présence métaphysique déjà annoncé, chercherait à mettre en évidence l'impossibilité, pour l'histoire, l'héritage et la tradition (et, donc, pour la philosophie), d'échapper au différer. Ruines et cendres s'esquisseraient ici comme des figures signifiant la revenance depuis toujours spectrale du passé, autrement dit l'impossible présentation d'un passé toujours déjà non présent à lui-même, comme les cendres de Celan. S'il y a témoignage d'un passé toujours passé, il serait empreint de cette spectralité, de cette prise de conscience de l'a-présentation, du fantasmatique, du spectre qui travaille tant le rapport au passé que la tension vers l'avenir. C'est ici que s'inscrit la réflexion derridienne de l'archive comme impensable totalisation et clôture de la mémoire, car «l'archive a lieu au lieu de défaillance originaire et structurelle de ladite mémoire» (1995:26).

La spectralité de la cendre ou de la ruine, de la trace ou de l'empreinte mnésique-anamnésique de l'histoire, de l'archive différentielle et de l'écriture, demanderait donc à être formulée non au présent - lequel effacerait l'absence logée dans toute spectralité -, mais au futur antérieur. Le temps de la cendre est un temps impossible qui se tient comme le futur d’un passé et le passé d'un futur: la cendre n'est ni n'est pas, elle n'a pas déjà été ni sera non plus dans un futur proche; la cendre derridienne aura été, répète incessamment le philosophe, elle aura toujours déjà été, elle aura toujours déjà été avant ce qu'elle incinère. C'est ainsi que la langue d'écrivain de ce philosophe fait arriver ce temps de l'impossible qui donne à penser une ruine avant la venue de cela même qu'elle ruine. D'un côté donc, la cendre aurait toujours eu lieu, tandis que, de l'autre, elle serait encore à venir, comme dans le poème de Paul Celan: «Soviel/ Zu segnende Asche» (1964), "Tant / de cendres à venir » (cité par Derrida, 1995 : 76). Par là, cette cendre rappelle autant qu'elle anticipe; elle ne peut rappeler que prenant par avance la place de ce qui n'est pas encore. Grâce à ce futur antérieur, Derrida libère la cendre de son rôle principal de porteparole, reste ou preuve d'un passé déjà révolu, pour lui donner la chance de la prophétie ou de l'annonciation.

Cette anticipation ruineuse, incinérée, se trouve à l'origine même de l'écriture et de la philosophie derridiennes. C'est bien le mot - donc l'écriture - qui est visé par Derrida dans les mots «cendre» et «ruine» dans Feu la cendre, auquel elles se substituent. Elles se substituent au mot par la métonymie, cette figure privilégiée dans la pensée derridienne: avant tout autre trope rhétorique, cette «cendre de nom figure» appellerait la «métonymie quand la cendre se sépare, une chose en figurant une autre dont il ne reste rien de figurable en elle»(Derrida, 1987: 55). Le renvoi et le jeu de différences propres à 
la trace font de la cendre une métonymie de la figuration différée de ce qui n'est plus figurable, car la cendre diffère de ce qu'elle nomme et est censée figurer.

Le figurable est renvoyé, différé, disséminé à jamais dans la cendre; c'est pourquoi une complication s'installe dès le moment où le philosophe, en 1990, dans Mémoires d'aveugle, déploie sa pensée du dessin, et plus spécifiquement de l'autoportrait, comme une ruine de la représentation.

On peut se demander ici : comment est-il possible de penser cette ruine originaire qui arrive à l'écriture - et dont Derrida aura dit qu'il n'y a « rien à toucher, aucune couleur, point de corps, des mots seulement » (ibid. : 55) - , comme ce qui assiste contemporainement la naissance du trait en dessin? Ou, pour le dire autrement, comment placer des cendres, ruines, traces ou marques, qui ne sont pas de l'ordre de la visibilité, au cœur même d'une réflexion sur le dessin qui fait partie des arts visuels, pour le catalogue du musée du Louvre Mémoires d'aveugle? Comment les y placer, de surcroît, quand, dans les dessins qui y sont rassemblés, la thématique ou la représentation de ruines au sens strict n'y est aucunement montrée?

Tout d'abord, le philosophe répond ainsi à cette espèce d'aporie concernant l'irreprésentable ou l'infigurable au cœur même du représentable et du figurable du dessin: la ruine ne serait pas à penser comme un thème ou une thématique, la ruine ne serait pas un sujet ou un objet à traiter, sur lequel on écrirait ou l'on dessinerait; elle serait bien plutôt la condition de toute écriture et de tout dessin, leur «condition de possibilité» ou leur «structure» la plus profonde. La ruine s'inscrirait dans une logique «transcendantale» («l'invisible condition de possibilité du dessin, le dessiner même, le dessin du dessin » qui «ne serait jamais thématique» [1990: 46]), par opposition à une logique «sacrificielle» thématique qui concernerait plutôt la représentation visuelle proprement dite (comme la figuration des ruines propre au XIXe siècle). $C^{\prime}$ est pourquoi, dans Mémoires d'aveugle, la pensée derridienne de la ruine engage non pas un discours esthétique ou historique sur les tableaux des paysages en ruine comme l'a pu faire Diderot à propos d'Hubert Robert, mais bien plutôt une réflexion sur l'impossibilité du dessin de soi ou autoportrait.

La ruine [en tant que ruine transcendantale] n'est pas devant nous, ce n'est ni un spectacle ni un objet d'amour. Elle est l'expérience même: ni le fragment abandonné mais encore monumental d'une totalité, ni seulement, comme le pensait Benjamin, un thème de la culture baroque. Ce n'est pas le thème, justement, cela ruine le thème, la position, la présentation ou la représentation de quoi que ce soit.

(Ibid.: 72)

Dans Mémoires d'aveugle, Derrida inscrit cette ruine a-thématique et a-présentative à l'origine de l'autoportrait de dessinateurs célèbres ou anonymes, genre qu'il préfère nommer en italien afin de souligner la non-coïncidence avec soi, ainsi que le retrait dans le trait du dessin: I'auto-ri-tratto italien rappelle bien l'origine d'un dessin qui ne serait qu'une simultanéité incontournable de trait (tratto) et de retrait (ri-tratto), dont l'équivalent littéral en français serait plutôt l'auto-retrait et non pas l'auto-por-trait, qui désigne sans hésitation l'autos, le soi, du portrait. Dès qu'il y aurait trait ou dépôt, un retrait s'avancerait déjà. Face donc au rêve d'identité et de coïncidence d'un modèle avec son image, d'un sujet avec son portrait, d'un soi-même avec un autoportrait, Derrida pointe la ruine du dessin de soi, ce qu'il aura appelé l'impossibilité d'une mimesis du même ou le "point d'autoportrait», le point de représentation du même. Le dessin se heurte à toute possibilité de figuration de soi car «l'hétérogénéité reste abyssale entre la chose dessinée et le trait dessinant, fût-ce entre une chose représentée et sa représentation, le modèle et l'image» (ibid.: 54). Mémoires d'aveugle serait à lire comme un autoportrait ruineux qui pense ensemble l'impossible spéculation ou réfléchissement visible et l'exercice de la pensée.

De même que la cendre qui ne garde en elle rien de figurable ou de visible en tant que tel, l'autoportrait derridien sera hanté par un certain aveuglement originaire qui continue à contester la présence métaphysique dans son apparaître. Dans son approche du visible et des arts visuels, le philosophe commence par rappeler l'arrière-fonds classique (indo-européen, grec et latin) qui rapporte les concepts de connaissance ou de savoir philosophique à la vision: «Idein, eidos, idea: toute I'histoire, toute la sémantique de l'idée européenne, dans sa généalogie grecque, on le sait, on le voit, assigne le voir au 
savoir » (ibid.: 18). Idein ou théorein, verbes grecs signifiant « voir », mais aussi « observer», "considérer», "réfléchir», " penser », «théoriser», "savoir», etc., sont ici des exemples de l'origine commune de la vision et du savoir dans le projet philosophique (Derrida rappelle la mythologie classique, mais aussi l'histoire de la philosophie «de Platon à Husserl»), qui aurait toujours supposé la présence à soi de la chose pour être vue et ensuite connue.

Dans cette perspective, le choix derridien de l'aveuglement serait donc à lire comme une contestation des philosophies de I'apparaître dans son rapport à la présence, laquelle ne tient évidemment pas compte du mouvement de la différance. C'est pourquoi, même si l'on pourrait croire paradoxale ou aporétique cette invisibilité de la ruine dans le contexte des dessins visibles de la collection du Louvre, elle concerne plus les présupposés d'un certain visible phénoménalisé dans la déconstruction de la lumière comme métaphore métaphysique, que l'expérience de l'œil lui-même (Derrida parlera d'un voir-toucher, de I'œil comme blessure, etc.)

Sur ce point, l'extrême cohérence de la philosophie derridienne fait que, depuis les premiers ouvrages en 1967 (La Voix et le Phénomène, De la grammatologie), l'impossible figuration et l'impossible visibilité de ce qu'il a appelé tout de suite la trace sont déjà annoncées. La trace derridienne, le trait, la cendre ou la ruine ne se rapportent aucunement au concept de forme (morphé mais aussi idea, eidos); c'est pourquoi ils arrivent à échapper à l'évidence ou à la présence. La trace de ces ruines n'est pas repérable, identifiable, sa présence est une absence, comme dans les pages blanches de Feu la cendre que le philosophe donne à lire comme des traces invisibles d'autres textes, incinérés, ruinés, qu'aucune marque ne pourra montrer ou signaler du doigt: «les envois totalement incinérés n'ont pu être indiqués d'aucune marque» (1987: 44).

C'est ainsi qu'il en arrivera à penser la ruine (sorte de paradigme de la non-forme ou de la non-présence) comme la structure invisible de l'œuvre, une ruine qui, loin de mettre en péril l'œuvre ou annoncer sa mort, «n'a jamais menacé le surgissement de l'œuvre» (1990: 69), car l'œuvre aura toujours compté, originairement, avec la ruine: il s'agit d'une ruine « qui ne vient pas après l'œuvre mais qui reste produite, dès l'origine, par l'avènement et la structure de l'œuvre » (ibid. : 68). Derrida arrivera ainsi à postuler une «ruine originaire» qui, loin de signifier «le vieillissement, l'usure, la décomposition anticipée ou cette morsure du temps dont un portrait souvent trahit l'appréhension», loin de «survenir comme un accident à un monument hier intact» (ibid.: 72), est une ruine contemporaine à la naissance de l'œuvre; elle habite les origines de la représentation.

Dans l'acheminement vers l'invisibilité constitutive de cette origine que le philosophe a appelée un «simulacre ruineux», la ruine est une «mémoire ouverte comme un œil ou la trouée d'une orbite osseuse qui vous laisse voir sans rien vous montrer du tout. Pour ne rien vous montrer du tout. "Pour" ne rien montrer du tout, c'est-à-dire à la fois parce que la ruine ne montre rien du tout, et en vue de ne rien montrer du tout. Rien de la totalité qui ne s'ouvre, se perce ou se troue aussitôt » (ibid.). La ruine ne se présente donc que comme trou ou œil ouvert qui rappelle une mémoire anamnésique et an-intentionnelle. La ruine n'aura jamais été pour ou en vue d'aucune monstration ou démonstration, son sens n'est pas celui de la preuve, de l'évidence ou de la présentation.

C'est par là que les phrases derridiennes «à l'origine il y eut la ruine» (ibid.: 69), "au début la ruine», auront voulu exprimer le désir d'une origine de la représentation et donc de l'écriture délivrée du rêve fantasmatique qui continue à penser la présence avant l'absence, le tout avant la partie, le plus avant le moins ou le monument avant la ruine.

D'où l' " amour » derridien des ruines. "Comment aimer autre chose que la possibilité de la ruine? Que la totalité impossible? L'amour a l'âge de cette ruine sans âge - à la fois originaire, infante même, et déjà vieille» (ibid.: 72).

RÉFÉRENCES BIBLIOGRAPHIQUES

DERRIDA, J. [1972] : «La différance», Marges de la philosophie, Paris, Minuit, 1-29; [1986] : Schibboleth, Paris, Galilée; [1987] : Feu la cendre, Paris, Éd. Des femmes; [1990] : Mémoires d'aveugle : I'Autoportrait et autres ruines, Paris, Éd. de la Réunion des musées nationaux; [1995] : Mal d'archive, Paris, Galilée.

Cixous, H. [1986] : «La Venue à l'écriture», Entre l'écriture, Paris, Éd. Des femmes. 